\title{
Joint Damage Accelerating Properties of Neutrophils
}

\author{
Viktoriya Milanova, Nina Ivanovska, Petya Dimitrova \\ Department of Immunology, Institute of Microbiology, Bulgarian Academy of Sciences, Sofia, Bulgaria \\ Email: petya_dimitrova@web.de
}

Received 10 April 2014; revised 10 May 2014; accepted 18 May 2014

Copyright (C) 2014 by authors and Scientific Research Publishing Inc.

This work is licensed under the Creative Commons Attribution International License (CC BY). http://creativecommons.org/licenses/by/4.0/

(c) (i) Open Access

\begin{abstract}
Neutrophils are innate immune cells involved in the initial inflammatory response and in the pathogenesis of rheumatoid arthritis (RA), an inflammatory joint disease. They produce cytokines, chemokines, proinflammatory mediators and secrete enzymes causing a direct destruction of cartilage and bone. Herein we investigated the ability of neutrophils to express the receptor activator of nuclear factor kappa-B ligand (RANKL) and to interfere with maturation of late pre-osteoclasts. The distribution of bone marrow (BM) Ly6G+ cells expressing RANKL was evaluated after BM cell dye labelling and transfer into zymosan-injected SCID recipient mice. Specific tartrate-resistant acid phosphatase (TRAP) staining was used to determine the number of multinucleated mature osteoclasts in the co-cultures of purified blood neutrophils with preosteoclasts. Ly6 G+ BM cells migrated extensively in synovial fluid and spleen of recipient zymosan-injected SCID mice. Labelled neutrophils have higher RANKL expression in synovial fluid unlike in spleen indicating that they obtained specific phenotype during their migration to the synovial fluid. Blood neutrophils increased the number of multinucleated mature osteoclasts in vitro. This effect was elicited by the pretreatment of neutrophils with interleukin (IL)-17. In summary, our study showed neutrophils's properties to accelerate joint damage via RANKL and interactions with osteoclasts.
\end{abstract}

\section{Keywords}

Neutrophils, RANKL, Cell Transfer, Zymosan, Osteoclasts

\section{Introduction}

Neutrophils play a key role in the perpetuation of inflammatory immune response in certain pathologic conditions such as rheumatoid arthritis. They acquire specific phenotype and functional state during disease development [1]. For example, neutrophils from RA patients highly express and secrete the pro-inflammatory cytokines 
tumor necrosis factor (TNF)- $\alpha$ and oncostatin M [2] [3]. They show enhanced inflammatory NF- $\kappa$ B (nuclear factor kappa-light-chain-enhancer of activated B cells) and STAT (signal transducer and activator of transcription) pathways and up-regulated Fc $\gamma$, complement and Toll-like (TLR) receptors, all delivering activation signals [4]. Recently, it has been shown that neutrophils can be a source of IL-17 [5], a key cytokine found in the synovial fluid and serum during the early stage of disease [6]. Indeed, arthritic neutrophils produce spontaneously IL-17 and their elimination with specific 1A8 antibody decreases IL-17 levels in both synovial fluid and blood [5]. Neutrophils from arthritic mice produce interferon (IFN)- $\gamma$ and show specific phenotype of double IFN- $\gamma+$ IL-17+ cells in synovial fluid and of single IFN- $\gamma+$ or IL-17+ cells in circulation [5]. In addition, neutrophils have prolonged half-life and secrete a variety of mediators that contribute to pain and tissue damage like prostaglandins, leukotrienes, oxygen species and proteolytic enzymes [4].

Recently, it has been found that neutrophils from RA patients express RANKL and RANK and secrete osteoprotegerin (OPG), a decoy receptor which competes with RANKL for RANK binding [7]. RA environment contributes to RANKL expression because the molecule is found on neutrophils from healthy donors incubated with RA serum [7]. In our previous study, we have detected RANKL expression on blood and synovial fluid neutrophils isolated from mice with zymosan-induced arthritis [5]. In vitro the surface RANKL is found on healthy mature mouse neutrophils from BM and circulation after incubation with exogenous IL-17 [5].

However, it is still unclear whether the migration of neutrophils bearing RANKL is directed specifically to synovial fluid. In the present study, we investigated the distribution of labelled neutrophils and their surface RANKL expression after transfer into recipient SCID mice. We also evaluated the ability of neutrophils to alter osteoclast maturation.

\section{Material and Methods}

\subsection{Mice and Knee Injection}

BALB/c and SCID (CB17 $\left.{ }^{\mathrm{TM}}\right)$ mice were purchased from the Charles River Laboratories. Mice were kept under standard conditions of a $12 \mathrm{~h}-12 \mathrm{~h}$ light-dark cycle and fed with a laboratory diet and water ad libitum. All experiments were approved by the Animal Care Committee at the Institute of Microbiology, Sofia in accordance with the National and International Guidelines. After anesthesia (sodium pentobarbital, $50 \mathrm{mg} / \mathrm{kg}$ and buprenorphinehydrochloride, $0.1 \mathrm{mg} / \mathrm{kg}$; intraperitoneal injection; both from Sigma-Aldrich, Munich, Germany) mice were injected at knee cavity with $10 \mu \mathrm{l}$ of zymosan suspension $(20 \mathrm{mg} / \mathrm{ml}$; Sigma-Aldrich, Munich, Germany) or $10 \mu 1$ of sterile phosphate-buffered saline (PBS; control group). Mice were monitored for knee swelling and redness at day 1 and 3 . The experiment was repeated 3 times.

\subsection{Neutrophil Purification and Flow Cytometry}

BM cells were collected from the tibia and femur of BALB/c mice. Exclusion dye staining with $0.05 \%$ Trypan blue showed more than $95 \%$ viable cells in isolated population. The cells were laid on gradient of $75 \%, 65 \%$, $55 \%$ Percoll in $0.15 \mathrm{M} \mathrm{NaCl}$ (GE Healthcare, Diegem, Belgium). Mature neutrophils from the $65 \% / 75 \%$ interface and the upper part of the $75 \%$ layer were harvested, washed and counted. Cell smears were prepared and Gimsa stained for evaluation of nuclear segmentation. Blood neutrophils were isolated from heparinized blood of BALB/c mice as previously described [8]. The population contains more than $95 \%$ viable cells.

$\mathrm{BM}$ and blood neutrophils were resuspended at concentration of $1 \times 10^{5} / \mathrm{ml}$ in $2 \%$ fetal calf serum (FCS)/ PBS/1 mm EDTA. They were stained with antibodies against Ly6G (clone 1A8) and CD11b (clone M1-70; both from BioLegend, Cambridge, UK) for 15 minutes. RANKL expression was determined after cell labelling with biotinylated anti-RANKL antibody (Abcam, London, UK) or isotype control (BioLegend, Cambridge, UK) for 20 minutes followed by avidin-FITC (BD Biosciences, San Diego, USA) staining for 15 minutes as previously shown [9]. The cell samples were subjected to flow cytometry analysis using BD LSR II cytometer with BD FACSDiva v6.1.2 Software (Becton Dickinson, San Jose, USA). BM and blood populations consist of 80\% $89 \%$ positive cells for Ly6G.

\subsection{Cell Transfer}

Freshly isolated BM Ly6G+ cells from BALB/c mice were resuspended at concentration $1 \times 10^{6} / \mathrm{ml}$ in warm PBS containing Cell Trace Violet dye $\mathrm{TM}^{\mathrm{M}}(2 \mu \mathrm{M}$; Invitrogen, Carlsbad, USA). The cells were incubated for 10 
minutes, $37^{\circ} \mathrm{C}$, in the dark. Any unbound dye was quenched by adding the same volume of FCS for 5 minutes. After washing with prewarmed $10 \%$ FCS/RPMI medium, neutrophils were resuspended in 5\% FCS/RPMI at concentration $1 \times 10^{9} / \mathrm{ml}$. SCID mice ( $\mathrm{n}=5 /$ group) were intravenously injected with $100 \mu \mathrm{l}$ of cell suspension ( 1 $\times 10^{8}$ cells/mice). At the time of cell transfer mice were injected in the knee cavity with zymosan or PBS as described above. At day 3 blood, BM, spleen and synovial fluid were collected. Cell populations were prepared and Violet dye+Ly6G+ cells were determined by flow cytometry.

\subsection{Osteoclast Differentiation and Maturation}

BM cells were collected, washed, counted and resuspended at $2 \times 10^{6} / \mathrm{ml}$ in $10 \%$ FCS/Minimum Essential Medium Eagle (MEM) medium (Lonza, Verviers, Belgium). They were incubated for 1 day with medium containing macrophage colony-stimulating factor (M-CSF; $100 \mathrm{ng} / \mathrm{ml}$; PeproTech, London, UK). Osteoclast precursors were generated in cultures with M-CSF (30 ng/ml) and RANKL (50 ng/ml; PeproTech, London, UK) [10]. After 3 days, fresh medium supplemented with growth factor M-CSF $(30 \mathrm{ng} / \mathrm{ml})$ and RANKL $(50 \mathrm{ng} / \mathrm{ml})$, IL-17 ((50 $\mathrm{ng} / \mathrm{ml}$, Abcam, London, UK) or blood neutrophils $\left(1 \times 10^{6} / \mathrm{ml}\right)$ were added to the cultures. The cells were let for 3 day maturation and specific TRAP staining was performed as previously described [10]. Numbers of nuclei in TRAP + cells were evaluated. In some experiments blood neutrophils from BALB/c mice were purified and resuspended at concentration of $1 \times 10^{6} / \mathrm{ml}$ in 10\% FCS $/$ RPMI containing IL-17 (40 ng/ml; Abcam, London, UK) for 24 hours, $37^{\circ} \mathrm{C}$. RANKL+ cells are enriched till $8 \%$ and OPG amounts are approximately $0.8 \mathrm{ng} / \mathrm{ml}$ in those cell cultures [5]. The cells were washed and added to osteoclast cultures.

\subsection{Statistical Analysis}

Results are expressed as the mean \pm SEM. Statistical significance of the data was determined with a two-tailed Student $t$ test using InStat3.0 and GraphicPad Prism software. $P$ values less than 0.05 were considered significant.

\section{Results}

\subsection{Cell Transfer of BM Ly6G+ Cells to Zymosan-Injected Recipient SCID Mice}

In order to study the homing of neutrophils expressing RANKL under inflammatory and normal conditions we have performed a set of experiments in which BM neutrophils were transferred to SCID mice at the time of zymosan knee injection (Figure 1). BM cells were isolated from healthy BALB/c mice and purified. The population consists of viable Ly6G+ cells expressing CD11b (Figure 1). Neutrophils were labelled with Violet dye at concentration of $2 \mu \mathrm{M}$ that failed to induce cell apoptosis (data not shown). The cells were transferred into SCID mice at concentration of $1 \times 10^{8}$ cells. At the same time SCID mice received knee injection of zymosan (ZY mice) and PBS. The distribution of neutrophils was determined among blood, BM, spleen and synovial fluid cells 3 days of post-zymosan injection. Violet dye+Ly6G+ cells were undetectable in blood probably due to their short half-life of several hours. We also evaluated the frequencies of the transferred cells in the marginated pools. Unlike in PBS injected group, Violet dye+ neutrophils migrated extensively to and accumulated in synovial fluid of zymosan-injected mice (Table 1). Labelled cells were detected in the PBS as well in ZY spleens but their frequencies were higher in ZY group (Table 1). Our data showed that Violet dye+ Ly6G+ cells in synovial fluid of ZY mice highly expressed RANKL, a molecule involved in osteoclastogenesis and bone destructive processes (Table 1). As surface RANKL was barely detectable on Violet dye+ spleen neutrophils we concluded that RANKL-bearing Ly6G+ cells traffic to synovial fluid rather than to other organs.

\subsection{Neutrophils Promote Pre-Osteoclast Maturation in Vitro}

RANKL-bearing neutrophils might contribute directly to the activation and maturation of osteoclasts.

Thus, we next evaluated the effect of neutrophils on osteoclast maturation. BM cells were isolated from $\mathrm{BALB} / \mathrm{c}$ mice and differentiated to late pre-osteoclasts as shown on schedule in Figure 2(A).

RANKL and M-CSF were able to induce osteoclast differentiation in a significant number of BM cells (Figure 2(A)-Figure 2(D)). At the last 3 days pre-osteoclasts were let to mature in the presence of fresh medium containing RANKL/M-CSF, RANKL/IL-17, M-CSF/neutrophils and M-CSF/neutrophils pretreated with IL-17. The latter Ly6G+ population was enriched till 8\% of RANKL+ cells (IL-17 pretreated group: 8.5\% \pm 
2.5\%; untreated neutrophil group: $1.8 \% \pm 0.2 \%, P<0.001$, Student $t$ test, see also [5]). The generated mature osteoclasts were recognized after specific TRAP staining and morphologically contained more than 4 nuclei (Figure 2(B)). The numbers of TRAP+ multinucleated cells dominated in the cultures with RANKL/ M-CSF (Figure 2(C)) while dinucleated TRAP+ preosteoclasts were observed in the IL-17/M-CSF group. The presence of neutrophils at the end stage of cultures resulted in the generation of mature osteoclasts similarly to RANKL/M-CSF group (Figure 2(C)). IL-17 pretreated neutrophils elicited osteoclast numbers (Figure 2(C)). Moreover osteoclasts in these cultures were with more than 5 nuclei (Figure 2(D)) suggesting that IL-17 enhanced the potential of neutrophils to promote osteoclast maturation.

\section{Discussion}

Neutrophils play a key role in the initiation of inflammation in joint inflammatory diseases like RA. They ac-

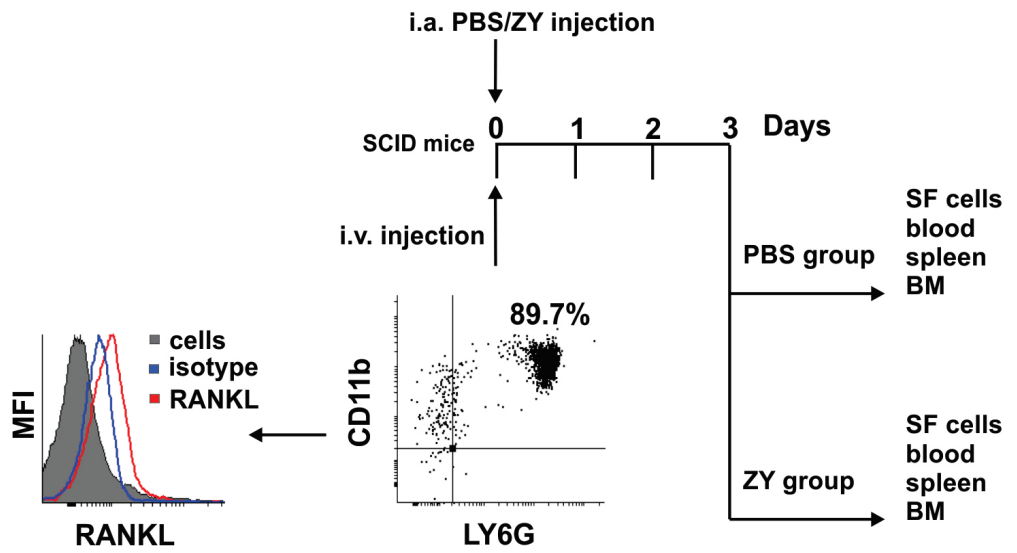

Figure 1. Transfer of Ly6G+CD11b+ cells to zymosan-injected SCID recipient mice. Ly6G+ CD11b+ cells were purified from BM of healthy BALB/c mice and then resuspended at concentration $1 \times 10^{6} / \mathrm{ml}$ in warm PBS containing Cell Trace Violet dye $\mathrm{T}^{\mathrm{TM}}(2 \mu \mathrm{M}$; invitrogen) as described in the Section Material and Methods. Cell population consisted of more than $95 \%$ viable cells, positive for Ly6G and expressing CD11b. Histogram shows bare expression of RANKL on BM neutrophils in comparison to isotype control staining (mean of fluorescent intensity: MFI). SCID mice ( $n=5 /$ group) were intravenously injected with $100 \mu$ of cell suspension $\left(1 \times 10^{8}\right.$ cells/mouse $)$. At the time of cell transfer mice were injected intraarticularly (i.a.) at the knee joint with zymosan (ZY group) or PBS (PBS group). On day 3 of cell transfer BM, blood, spleen and synovial fluid (SF) cells were collected and subjected to flow cytometry analyses. The experiment was repeated 3 times.

Table 1. Distribution of Ly6G+ cells after their transfer in zymosan-injected recipient SCID mice.

\begin{tabular}{|c|c|c|c|c|c|}
\hline Groups & Distribution & BM & Spleen & Blood & Synovial fluid \\
\hline \multirow{3}{*}{ PBS group } & $\begin{array}{l}\text { Ly6G }+ \text { cells } \\
(\% \text { total })\end{array}$ & $56 \pm 9$ & $20.4 \pm 9.5$ & $4.8 \pm 1.0$ & $0.04 \pm 0.01$ \\
\hline & $\begin{array}{c}\text { Violet }+ \text { cells } \\
(\% \text { of Ly6G+ cells })\end{array}$ & $0.58 \pm 0.01$ & $10.5 \pm 2.4$ & $0.00 \pm 0.00$ & $0.0 \pm 0.0$ \\
\hline & $\begin{array}{c}\text { RANKL } \\
\text { (MFI/Violet+ Ly6G+ cells) }\end{array}$ & $214 \pm 56$ & $105 \pm 35$ & $98 \pm 12$ & $87 \pm 14$ \\
\hline \multirow{3}{*}{ ZY group } & Ly6G+ cells (\% total) & $74 \pm 12$ & $41.8 \pm 8.2^{*}$ & $10.5 \pm 2.5^{*}$ & $4.52 \pm 0.98^{*}$ \\
\hline & $\begin{array}{c}\text { Violet }+ \text { cells } \\
(\% \text { of Ly6G+ cells })\end{array}$ & $0.67 \pm 0.03$ & $21.4 \pm 9.7^{*}$ & $0.71 \pm 0.07$ & $65.2 \pm 8.9^{*}$ \\
\hline & $\begin{array}{c}\text { RANKL } \\
\text { (MFI/Violet+ Ly6G+ cells) }\end{array}$ & $231 \pm 116$ & $124 \pm 44$ & $108 \pm 54$ & $214 \pm 22^{*}$ \\
\hline
\end{tabular}

a.Ly6G+CD11b+ cells were purified and transferred as described in Figure 1. Data represent the mean \pm SEM from 3 different experiments involving 5 mice per group. ${ }^{*} P<0.05$ when compared PBS to ZY group. 
A.
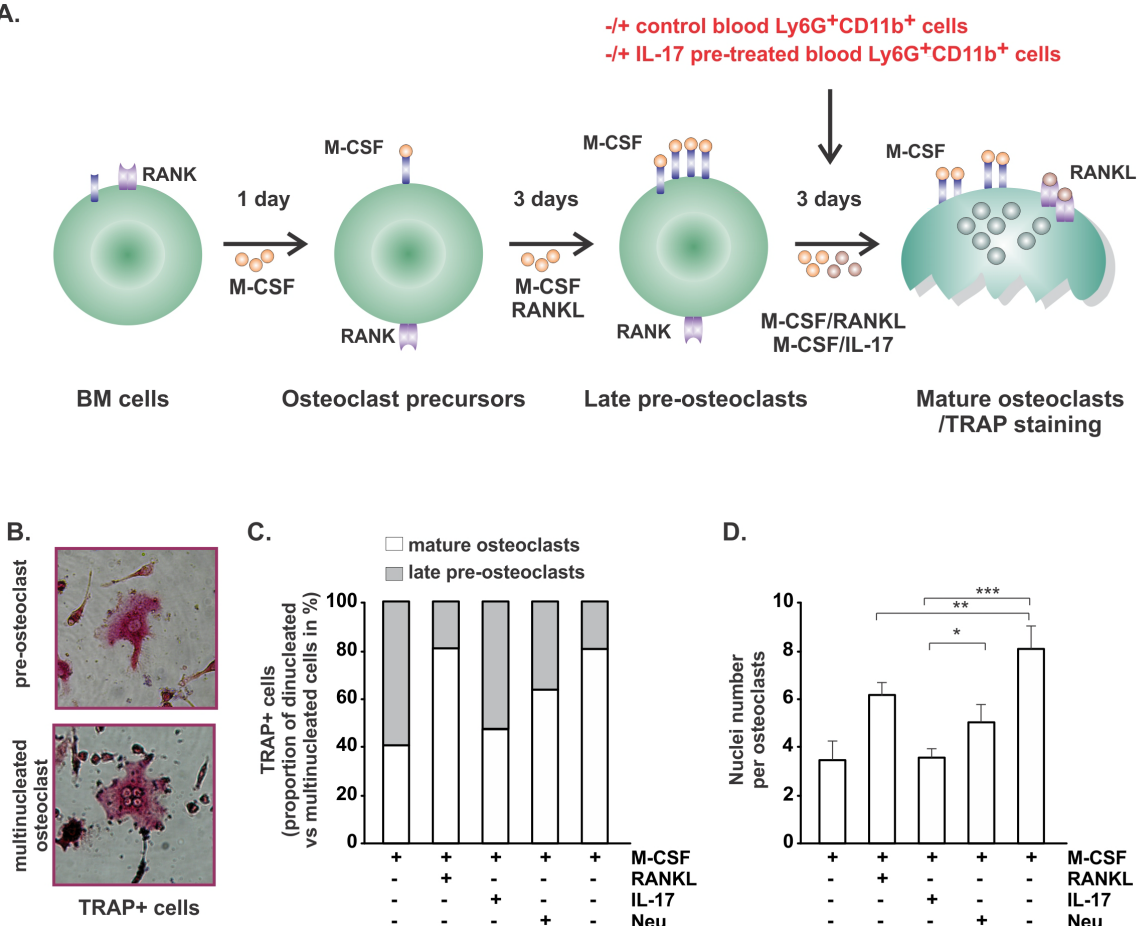

C.

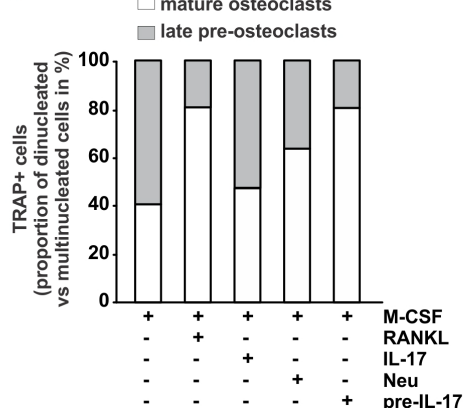

D.



Figure 2. Osteoclast maturation in the presence of blood neutrophils. A) Schedule of osteoclast differentiation and maturation in vitro. BM cells were collected and incubated for 1 day with medium containing M-CSF. Osteoclast precursors were generated in the cultures with M-CSF/RANKL. After 3 days fresh medium supplemented with growth factors M-CSF/ RANKL or M-CSF/IL-17, M-CSF/blood neutrophils $\left(1 \times 10^{6} / \mathrm{ml}\right)$ were added to the cultures. In some experiments neutrophils were pretreated with IL-17 as described in the Section of Material and Methods. Osteoclast precursors were let for 3 day maturation and specific TRAP staining was performed; B) Representative TRAP staining showing preosteoclast and multinucleated mature osteoclast; magnification $1 \times 100$; C) The proportion of dinucleated preosteoclasts to multinucleated mature osteoclasts was calculated for each well/sample in one representative experiment of three, involving 5 mice per group; D) Number of nuclei of TRAP+ cells indicative for mature phenotype. Data in C) and D) represent the mean \pm SEM from 3 different experiments involving 5 mice per group. ${ }^{* * *} P<0.001,{ }^{* *} P<0.01,{ }^{*} P<0.05$, Student $t$ test.

cumulate in the synovial fluid inducing a perpetuation of immune response via secretion of oxygen species, prostaglandins, leukotrienes and wide variety of proteolytic enzymes. Zymosan, a TLR2 ligand, triggers acute and chronic inflammation after intraperitoneal and intraarticular injections [11] [12]. Blood and synovial neutrophils from mice with zymosan-induced arthritis are in primed state and up-regulate TLR2 [5] [11] [12]. The engagement of TLR2 induces neutrophil migration via activation of ERK and p38 MAPK pathways [13]. Our study showed that zymosan knee injection promoted homing of transferred Violet dye+ neutrophils in spleen and migration in synovial fluid. Despite that the frequencies of Ly6G+ cells increased in blood of zymosan-injected mice, we failed to detect labelled neutrophils after 3 days at that location probably because of their short half-life. It has been shown that granulocytes disappear from circulation within 7.2 hours after their transfer in SCID mice and the recovery of the total granulocyte pool occurs within 3 days [14]-[16]. Concerning spleen, Violet dye+ neutrophils can be located in the red pulp and the marginal zone. In human, splenic neutrophils produce B cell stimulating factor (BAFF), a proliferation inducing ligand (APRIL) and IL-21 and stimulate antibody production and somatic hypermutation of marginal zone B cells [17]. The splenic pool of neutrophils is formed after birth in a result of continuous stimulation of circulating cells by microbial products. TLR2 ligand knee injection increased Ly6G+ reservoir unlike in PBS group. This process might be involved chemokine receptor CXCR4 and integrin CD11b which are sensitive to TLR2 stimulation and are able to regulate neutrophil 
retention [18]. However, more investigations are required to understand the function of the neutrophils in the splenic pool during chronic joint inflammatory conditions.

We found that Violet dye+ neutrophils migrated to the synovium of zymosan injected mice. Neutrophil recruitment can be regulated via TLR2 and its interaction with S100A9 (known as migration inhibitory factor-related protein 14 (MRP-14) and CXCR2 [18] [19]. By contrast to splenic neutrophils, synovial Violet+ Ly6G+ cells expressed RANKL. The data confirmed our previous observation for increased frequencies of RANKL+ neutrophils in synovial fluid of mice with zymosan-induced arthritis. It is more likely that Ly6G+ cells obtained this phenotype during trafficking and locally in result of altered environment. Injected at the knee cavity, TLR2 ligand can trigger cytokine (IL-6, IL-8, TNF- $\alpha$, IL-1, IL-17) and chemokine (macrophage inflammatory protein (MIP)-1 $\alpha$, monocyte chemoattractant protein (MCP)-1) production by fibroblasts, synoviocytes and immune cells. All these factors drive neutrophil chemotaxis and recruitment but can have an impact on RANKL expression as well. Correspondingly, synovial fluid from RA patients increased RANKL expression on healthy blood neutrophils unlike that from healthy donors [7]. It has been shown that TNF, via TNF type 1 receptor (TNFR1) can stimulate RANKL-induced mediators, including c-Src, TNF receptor-associated factor (TRAF)-2, TRAF-6, and MEKK-1 [20]. The recently identified tumor necrosis factor-like weak inducer of apoptosis (TWEAK) in synovial fluid of RA patients and mice with collagen-induced arthritis is able to induce RANKL expression on joint cells [21]. In our previous study, we have demonstrated that IL-17 is a key factor for RANKL expression on neutrophils [5]. We find that the cytokine can interfere with RANKL directly or indirectly via OPG secretion. In cultures, exogenous IL-17 induces RANKL expression on healthy blood neutrophils and BM Ly6G+ cells and up-regulates surface RANKL on arthritic blood neutrophils along with an inhibition of OPG secretion [5]. These effects of IL-17 can be regulated by the engagement of TLR2 [5]. In the present study, RANKL expression on neutrophils is found very early after TLR2 injection (day 3) when bone resorption is still milder. Thus, we built the hypothesis that RANKL-bearing neutrophils in synovial fluid might be important for the initiation of bone resorption. This process involves the maturation and activation of osteoclasts degrading collagen and proteoglycans via production of matrix metalloproteinase. Osteoclast differentiation and maturation is driven directly by RANKL and indirectly by the decoy receptor OPG. Under physiological conditions, RANKL is expressed by stromal cells and osteoblasts in BM. However, under inflammatory conditions, immune cells like $\mathrm{T}$ cells, monocytes and neutrophils can express membrane bound RANKL. The molecule is cleaved from the membrane surface by matrix metalloproteinase and accumulates in extracellular matrix, synovial fluid and serum. The level of soluble RANKL as well as RANKL/OPG axis is indicative for severity of bone damage and disease [22].

In vitro RANKL drives osteoclastogenesis [10] [22]. We found that bone marrow cells differentiated to preosteoclasts for 3 days in the presence of RANKL. According to this in vitro system, the cells undergo maturation for next 3 days of culture and finally are recognized as specific multinucleated TRAP positive cells. There is a positive correlation between the number of nuclei per osteoclast, their ability to form pits on dentine slides and efficiency to resorb bone matrix [23]. We found that the numbers of TRAP+ multinucleated cells dominated in the cultures with RANKL/M-CSF and M-CSF/neutrophils. Indeed IL-17 pretreated neutrophils elicited the formation of osteoclasts and numbers of their nuclei. We thought that IL-17 enhanced the potential of neutrophils to promote osteoclast maturation via up-regulation of RANKL expression and an increase in the numbers of RANKL+ cells. Multinucleated osteoclasts can originate from fused RANK+ mononuclear precursors after their contact to RANKL-bearing neutrophils. RANKL/RANK interaction triggers the expression of fusionmediating molecules such as $\mathrm{d} 2$ isoform of vacuolar ATPase Vo domain (Atp6v0d2) and the dendritic cellspecific transmembrane protein (DC-STAMP) [24]. It is also possible that neutrophils deliver additional signals via adaptor proteins containing immunoreceptor activating motif (ITAM) such as DAP12, and Fe $\gamma \mathrm{R}$ [25].

In vivo RANKL-bearing neutrophils can interact with mononuclear precursors recruited into synovial fluid. Ly6G+ RANKL+ cells can provide a regulatory loop via the expression of chemokine (CCR2 and CCR4), G protein-coupled receptors and PI3K signaling pathways [26], all important for acquiring the multinucleated phenotype. It has been shown that RANKL/RANK interaction induces MCP-1 secretion and stimulates TRAP and calcitonin receptor via NF-ATc transcription factor [27]. The interaction between neutrophils and osteoclasts has been visualized by confocal laser microscopy [28]. Indeed it has been shown that RANKL-associated activation of neutrophils is mediated via the tyrosine phosphorylation of Src-homology domain-containing cytosolic phosphatase 1 and subsequent alteration of the cytokine production with impact on osteoclasts [28]. 


\section{Conclusion}

In summary, our study showed that RANKL-bearing neutrophils were found in synovial fluid very early after the initiation of knee inflammation but failed to populate and replenish the marginated splenic pool. Neutrophils were able to increase the osteoclast maturation of preosteoclasts and this effect could be elicited by IL-17 pretreatment. These data indicated that novel agents limiting bone resorption might be based on interventions inhibiting the migration and numbers of RANKL-bearing neutrophils.

\section{Acknowledgements}

The work was financially supported by Inter-Pasteurien Concerted Actions Grant A05_11, France and by grant 183/23551 from the Program BG051PO001/3.3-05-0001 of Bulgarian Ministry of Education and Science and European Social Fund. V.M. and P.D. were involved in the acquisition of the data. P.D. designed the research and study conception. V.M., P.D, N.I. were involved in drafting the article and revising it critically for the important intellectual content. All authors approved the final version to be published. V.M. is a PhD student at the Department of Immunology, Institute of Microbiology, Sofia, Bulgaria and this work will be a part of her PhD thesis.

\section{References}

[1] Cascão, R., Rosário, H.S., Souto-Carneiro, M.M. and Fonseca, J.E. (2010) Neutrophils in Rheumatoid Arthritis: More than Simple Final Effectors. Autoimmunity Reviews, 9, 531-535. http://dx.doi.org/10.1016/j.autrev.2009.12.013

[2] Cross, A., Edwards, S.W., Bucknall, R.C. and Moots, R.J. (2004) Secretion of Oncostatin M by Neutrophils in Rheumatoid Arthritis. Arthritis \& Rheumatism, 50, 1430-1436. http://dx.doi.org/10.1002/art.20166

[3] Wright, H.L., Chikura, B., Bucknall, R.C., Moots, R.J. and Edwards, S.W. (2011) Changes in Expression of Membrane TNF, NF- $\kappa$ B Activation and Neutrophil Apoptosis during Active and Resolved Inflammation. Annals of the Rheumatic Diseases, 70, 537-543. http://ard.bmj.com/content/early/2010/11/24/ard.2010.138065.abstract http://dx.doi.org/10.1136/ard.2010.138065

[4] Wright, H.L., Moots, R.J., Bucknall, R.C. and Edwards, S.W. (2010) Neutrophil Function in Inflammation and Inflammatory Diseases. Rheumatology, 49, 1618-1631.

http://rheumatology.oxfordjournals.org/content/49/9/1618.abstract http://dx.doi.org/10.1093/rheumatology/keq045

[5] Milanova, V., Ivanovska, N. and Dimitrova, P. (2014) TLR2 Elicits IL-17-Mediated RANKL Expression, IL-17, and OPG Production in Neutrophils from Arthritic Mice. Mediators of Inflammation, 2014, 14. http://dx.doi.org/10.1155/2014/643406

[6] Raza, K., Falciani, F., Curnow, S.J., Ross, E.J., Lee, C.Y., Akbar, A.N., Lord, J.M., Gordon, C., Buckley, C.D. and Salmon, M. (2005) Early Rheumatoid Arthritis Is Characterized by a Distinct and Transient Synovial Fluid Cytokine Profile of T Cell and Stromal Cell Origin. Arthritis Research \& Therapy, 7, R784-R795.

http://arthritis-research.com/content/7/4/R784 http://dx.doi.org/10.1186/ar1733

[7] Poubelle, P.E., Chakravarti, A., Fernandes, M.J., Doiron, K. and Marceau, A.A. (2007) Differential Expression of RANK, RANK-L, and Osteoprotegerin by Synovial Fluid Neutrophils from Patients with Rheumatoid Arthritis and by Healthy Human Blood Neutrophils. Arthritis Research \& Therapy, 9, R25. http://dx.doi.org/10.1186/ar2137

[8] Boxio, R., Bossenmeyer-Pourie, C., Steinckwich, N., Dournon, C. and Nusse, O. (2004) Mouse Bone Marrow Contains Large Numbers of Functionally Competent Neutrophils. Journal of Leukocyte Biology, 75, 604-611. http://www.jleukbio.org/content/75/4/604.abstract http://dx.doi.org/10.1189/jlb.0703340

[9] Dimitrova, P., Ivanovska, N., Belenska, L., Milanova, V., Schwaeble, W. and Stover, C. (2012) Abrogated RANKL Expression in Properdin-Deficient Mice Is Associated with Better Outcome from Collagen-Antibody-Induced Arthritis. Arthritis Research \& Therapy, 14, R173. http://arthritis-research.com/content/14/4/R173 http://dx.doi.org/10.1186/ar3926

[10] Takahashi, N., Udagawa, N., Kobayashi, Y. and Suda, T. (2007) Generation of Osteoclasts in Vitro, and Assay of Osteoclast Activity. Methods of Molecular Medicine, 135, 285-301. http://dx.doi.org/10.1007/978-1-59745-401-8 18

[11] Dimitrova, P., Ivanovska, N., Schwaeble, W., Gyurkovska, V. and Stover, C. (2010) The Role of Properdin in Murine Zymosan-Induced Arthritis. Molecular Immunology, 47, 1458-1466. http://dx.doi.org/10.1016/j.molimm.2010.02.007

[12] Ivanovska, N.D., Dimitrova, P.A., Luckett, J.C., El-Rachkidy Lonnen, R., Schwaeble, W.J. and Stover, C.M. (2008) 
Properdin Deficiency in Murine Models of Nonseptic Shock. Journal of Immunology, 180, 6962-6969. http://dx.doi.org/10.4049/jimmunol.180.10.6962

[13] Aomatsu, K., Kato, T., Fujita, H., Hato, F., Oshitani, N., Kamata, N., Tamura, T., Arakawa, T. and Kitagawa, S. (2008) Toll-Like Receptor Agonists Stimulate Human Neutrophil Migration via Activation Of Mitogen-Activated Protein Kinases. Immunology, 123, 171-180. http://www.jimmunol.org/content/180/10/6962.abstract

[14] Arnardottir, H., Freysdottir, J. and Hardardottir, I. (2012) Two Circulating Neutrophil Populations in Acute Inflammation in Mice. Inflammation Research, 61, 931-939. http://dx.doi.org/10.1007/s00011-012-0484-0

[15] Cartwright, G.E., Athens, J.W. and Wintrobe, M.M. (1964) Analytical Review: The Kinetics of Granulopoiesis in Normal Man. Blood, 24, 780-803. http://bloodjournal.hematologylibrary.org/content/24/6/780.abstract

[16] Summers, C., Rankin, S.M., Condliffe, A.M., Singh, N., Peters, A.M. and Chilvers, E.R. (2010) Neutrophil Kinetics in Health and Disease. Trends in Immunology, 31, 318-324. http://dx.doi.org/10.1016/j.it.2010.05.006

[17] Cerutti, A., Puga, I. and Magri, G. (2013) The B Cell Helper Side of Neutrophils. Journal of Leukocyte Biology, 94, 677-682. http://www.jleukbio.org/content/94/4/677.abstract http://dx.doi.org/10.1189/jlb.1112596

[18] Eash, K.J., Greenbaum, A.M., Gopalan, P.K. and Link, D.C. (2010) CXCR2 and CXCR4 Antagonistically Regulate Neutrophil Trafficking from Murine Bone Marrow. Journal of Clinical Investigation, 120, 2423-2431. http://dx.doi.org/10.1172/JCI41649

[19] Moles, A., Murphy, L., Wilson, C.L., Chakraborty, J.B., Fox, C., Park, E.J., Mann, J., Oakley, F., Howarth, R., Brain, J., Masson, S., Karin, M., Seki, E. and Mann, D.A. (2014) A TLR2/S100A9/CXCL-2 Signaling Network Is Necessary for Neutrophil Recruitment in Acute and Chronic Liver Injury in the Mouse. Journal of Hepatology, 60, 782-791. http://dx.doi.org/10.1016/j.jhep.2013.12.005

[20] Takada, Y. and Aggarwal, B.B. (2004) Evidence That Genetic Deletion of the TNF Receptor p60 or p80 in Macrophages Modulates RANKL-Induced Signaling. Blood, 104, 4113-4121. http://bloodjournal.hematologylibrary.org/content/104/13/4113.abstract http://dx.doi.org/10.1182/blood-2004-04-1607

[21] Park, J.-S., Kwok, S.-K., Lim, M.-A., Oh, H.-J., Kim, E.-K., Jhun, J.-Y., Ju, J.H., Park, K.-S., Park, Y.-W., Park, S.-H., Kim, H.-Y., Cho, Y.-G. and Cho, M.-L. (2013) TWEAK Promotes Osteoclastogenesis in Rheumatoid Arthritis. American Journal of Pathology, 183, 857-867. http://dx.doi.org/10.1016/j.ajpath.2013.05.027

[22] Wada, T., Nakashima, T., Hiroshi, N. and Penninger, J.M. (2005) RANKL-RANK Signaling in Osteoclastogenesis and Bone Disease. Trends in Molecular Medicine, 12, 17-25. http://dx.doi.org/10.1016/j.ajpath.2013.05.027

[23] Piper, K., Boyde, A. and Jones, S.J. (1992) The Relationship between the Number of Nuclei of an Osteoclast and Its Resorptive Capability in Vitro. Anatomy and Embryology, 186, 291-299. http://dx.doi.org/10.1007/BF00185977

[24] Lee, S.H., Rho, J., Jeong, D., Sul, J.Y., Kim, T., Kim, N., Kang, J.S., Miyamoto, T., Suda, T., Lee, S.K., Pignolo, R.J., Koczon-Jaremko, B., Lorenzo, J. and Choi, Y. (2006) v-ATPase V0 Subunit d2-Deficient Mice Exhibit Impaired Osteoclast Fusion and Increased Bone Formation. Nature Medicine, 12, 1403-1409. http://dx.doi.org/10.1038/nm1514

[25] Humphrey, M.B., Ogasawara, K., Yao, W., Spusta, S.C., Daws, M.R., Lane, N.E., Lanier, L.L. and Nakamura, M.C. (2004) The Signaling Adapter Protein DAP12 Regulates Multinucleation during Osteoclast Development. Journal of Bone \& Mineral Research, 19, 224-234. http://dx.doi.org/10.1359/JBMR.0301234

[26] Haringman, J.J., Ludikhuize, J. and Tak, P.P. (2004) Chemokines in Joint Disease: The Key to Inflammation? Annals of the Rheumatic Diseases, 63, 1186-1194. http://ard.bmj.com/content/63/10/1186.abstract http://dx.doi.org/10.1136/ard.2004.020529

[27] Bar-Shavit, Z. (2007) The Osteoclast: A Multinucleated, Hematopoietic-Origin, Bone-Resorbing Osteoimmune Cell. Journal of Cellular Biochemistry, 102, 1130-1139. http://dx.doi.org/10.1002/jcb.21553

[28] Chakravarti, A., Raquil, M.A., Tessier, P. and Poubelle, P.E. (2009) Surface RANKL of Toll-Like Receptor 4-Stimulated Human Neutrophils Activates Osteoclastic Bone Resorption. Blood, 114, 1633-1644. http://dx.doi.org/10.1182/blood-2008-09-178301 